\title{
CRISTINA TRIVULZIO BELGIOJOSO Y SU IDEA DE ESTADO: UNA PATRIOTA POLITÓLOGA ${ }^{1}$
}

\author{
Silvio $\operatorname{Cosco}^{2}$
}

\section{Cristina Trivulzio Belgiojoso y su idea de estado: una patriota politóloga}

Resumen: Cristina Trivulzio Belgiojoso es una patriota italiana del siglo XIX. Escritora, periodista, y activista, participa activamente en movimiento que lleva a la unificación italiana en el 1861. En su ensayo, describe su sueño de una nación más fuerte, justa, culta y solidaria. En un periodo de plena éxtasis celebrativa y patriótica, ella supo analizar lucidamente los defectos de la nueva Italia.

Palabras clave: Cristina Trivulzio Belgiojoso, patriota, politóloga, Unificación italiana.

Cristina Trivulzio Belgiojoso and her idea of state: A patriot political scientist Abstract: Cristina Trivulzio Belgiojoso is a 19th century Italian patriot, writer, journalist and activist who actively participates in the Italian unification in 1861. In her essay, she describes her dream of a strong, fair, educated and caring nation. In a time of patriotic ecstasy, she knew lucidly how to analyze the defects of the new Italy.

Keywords: Cristina Trivulzio Belgiojoso, patriot, political scientist, Italian unification.

Propagandistas, escritoras, poetas, conspiradoras, educadoras, enfermeras, combatientes, entre las mujeres que participaron en, y contribuyeron a, la unificación italiana de 1861, hay una que merece una mención especial, también por su difícil clasificación $^{3}$, es Cristina Trivulzio di Belgiojoso, nacida en Milán en 1808, en el seno de una familia de la alta aristocracia. Su contribución a la causa del Resurgimiento tuvo repercusión en todos los sectores de la vida pública en diferentes zonas de Italia ${ }^{4}$. Fue promotora de la unidad nacional al tiempo que defensora de los derechos de las mujeres.

\footnotetext{
${ }^{1}$ Fecha de recepción: 15/10/2016.

Fecha de aceptación: 20/12/2016.

2 Asistente Honorario, Departamento de Filologías Integradas, Universidad de Sevilla; $\square$ silviocosco@libero.it.

${ }^{3}$ Véase sobre las patriotas italianas Barbiera, Raffaello. 1923. Italiane gloriose, Milano: Vallardi; Cepeda Fuentes, Marina. 2011. Sorelle d'Italia. Le donne che hanno fatto il risorgimento, Torino: Blu; Comba, Eugenio. 1935. Donne italiane illustri, Torino: Paravia.

${ }^{4}$ Sobre la vida de Cristina Trivulzio di Belgiojoso, véase: La principessa Belgiojoso. I suoi amici e nemici, il suo tempo, Milano: Treve, 1902; Passioni del Risorgimento. Nuove pagine sulla principessa Belgiojoso e il suo tempo, Milano; Rossi, Mino, 1903; y Cristina Trivulzio, principessa di Belgiojoso. Il pensiero politico, Passirano (Br): Franciacorta, 2005.
} 
Su labor fue variada e ingente: recibió en su salón a los patriotas, financió sus empresas con fondos que procuró incesantemente y publicó "algunos trabajos donde vibraba el sentimiento dominante, el de la independencia italiana $[\ldots]$ y $[\ldots]$ sus escritos perseguidos por Austria, animaron a los jóvenes a combatir" (Angeloni 1911: 115). En el punto de mira de los austriacos, dejó Milán para refugiarse primero en Génova y luego en París, donde recibió a exiliados italianos.

"Enviadme a Italia a hacer propaganda, sabré disfrazarme de forma que nadie me conozca, tengo una vieja deuda con Austria, formé un grupo de soldados la otra vez, esta vez combatiré de persona" (Arriaga Flórez 2011: 7). Con estas palabras incendiarias, en 1831, Cristina Trivulzio de Belgioioso, solicita a Mazzini, patriota y entre los primeros conspiradores para la unificación italiana, que la deje volver a Italia para combatir contra la ocupación austriaca.

A su casa, centro cultural de la época, acudieron, entre otros, Lizt y Rossini mientras se conspiraba para la unificación italiana. En 1845 fundó La gazzetta italiana, que enviaba de contrabando, a través la cual difundía los ideales libertarios. En 1848, en Nápoles, formó el batallón de voluntarios que condujo hasta a Milán donde entró triunfalmente, agitando la bandera tricolor; en Roma en 1848, defendiendo la Republica, fue responsable de los hospitales ambulantes.

Tras el cierre de la Gazzetta, funda L'Ausonio, una revista mensual de más de ochenta páginas. Desde sus paginas, Cristina aclaró las intenciones que la movián:

Esta publicación está destinada a hacer conocer a los italianos y a los extranjeros las condiciones de nuestro territorio. Para encontrar remedio a las heridas primero es necesario exponerlas y hacer un cuadro exacto y sucinto del estado actual de Italia bajo todos los aspectos, es decir, una exposición de las condiciones morales, políticas, administrativas, financieras de este país. (Gasparinetti, 1930: 103)

Tan importante fue su contribución a la causa italiana, que la santurrona historia patriarcal le perdonó la vida privada. "Concorde al marido en la política, no lo era en las cosas domésticas, de ahí la separación conyugal", recordaba el historiador Angeloni (1911: 12). Tampoco fue exaltada, por aquella época, ni su consciencia de género ni su 
labor a favor de la emancipación de las mujeres. Autora del admirable De la presente condición de las mujeres y de su futuro en 1866, observó un gradual cambio en la percepción de las mujeres "extraordinarias" por parte de los hombres, por lo cual invitó a sus congéneres femeninas "a actuar de tal manera que estas excepciones se vuelvan más numerosas, hasta que el respeto tributado a algunas de ellas repercuta y se extienda gradualmente a todo el sexo femenino" (Arriaga Florez 2011: 29). Cristina tuvo el mérito de ponerse como ejemplo de mujer consciente de la necesidad de luchar por una liberación del género femenino.

En el 1861 se logró, por fin, la unidad política de Italia que tanto deseaba, por lo que optó por dejar la política con cierta serenidad. Vivió sus últimos años en Lombardía, entre Milán y el lago de Como. Cuando murió en 1871, a los 63 años, ningún político de Italia, país que ella tan generosamente había contribuido a unir, asistió a su funeral. Su compromiso multifacético produjo cierto desapunto e insatisfacción entre las diferentes personalidades de la época. Vincenzo Gioberti, patriota de la facción católica, dijo sobre Cristina que era una mujer que había olvidado "la reserva e verecundia" (Gioberti 1911: 18). Alessandro Manzoni, escritor de referencia del "800 italiano, criticaba la manía de “esa señora" de difundir el acceso a la educación entro los ciudadanos ${ }^{1}$. Y Alessandro Luzio, la describía como una impulsiva de sensaciones violentas y de contrastes románticos, deseosa de celebridad y amante de las fiestas ${ }^{2}$. Como sostenía Aldobrandino Malvezzi, Cristina reunía en sí dos tipos de mujeres que normalmente se consideran incompatibles: "su característica más genial es la de saber ser al mismo tiempo una mujer de mundo, que nos describen llena del más sutil e insinuante encanto femenino y mujer fuerte, viril, seria, equilibrada, dotada de sentido práctico y decidida en la acción, cuando se ocupaba de cuestiones políticas" (Malvezzi 1936: 131).

Como muchas figuras femeninas de este periodo, la princesa también aparece como ejemplo de abnegación patriótica en retratos idealizados, que ignoran la persona de carne y hueso para convertirla en un icono de la nación, como el que nos ofrece La Centuria di donne illustri italiane: "Literata, mujer de ciencia, gran escritora y patriota

\footnotetext{
${ }^{1}$ Cit. in Severgnini, Luigi, 1972. La principessa di Belgiojoso, Milano: Virgilio.

${ }^{2}$ Véase Luzio, A, in Cristina Belgioioso, in "Il corriere della Sera", Milano, 3 agosto 1902.
} 
incomparable...que por bien de los demás y del propio país se sacrificó a sí misma, y su propia sustancia" (AA.VV., 1883: 12).

Si bien ayudó a sus compañeros varones en múltiples hazañas para lograr el sueño de la Independencia de Italia, su lucidez y su objetividad la condujo a criticarles en los albores del nuevo país. Al margen de una serie de voces corales surgidas para celebrar la nueva retórica de la patria, Cristina Trivulzio di Belgiojoso, escribió en 1868 el ensayo Osservazioni sullo stato attuale dell'Italia e sul suo avvenire. En él observamos a una patriota que no deja de ser una ciudadana, parte de una comunidad, con un fuerte sentido crítico de naturaleza propositiva. En él evidencia los problemas a los que se debe enfrentar la recién nacida nación italiana y propone posibles soluciones ${ }^{3}$ para los mismos. Cristina tiene 60 años. Según Maldini Chiarito es:

la edad de los balances, públicos y privados, de la añorancia, de lo que se ha obtenido y perdido, de lo que se podía hacer, de los fracasos, de la nueve pruebas de enfrontar [...] y para Cristina, que había contribuido con muchas energías a la trabajosa realización del primer Resurgimiento, es importante continuar a medirse también con los problemas del periodo pos unitario, de los cuales intuye la grandeza. (Maldini Chiarito: 2010: 200)

Cristina está totalmente al margen de ese vacuo coro patriótico que afirma: "hemos creído demasiado ingenuamente que, después de las victoria del "59 y del "60, nuestras cuestiones progresarían por sí mismas, sin desviarse nunca, y sin que nadie se tomara la responsabilidad de guiarlas" (Trivulzio 1868: 146) ${ }^{4}$. Sin embargo, se distancia también del coro de los descontentos con tonos que para la estudiosa Maldini Chiarito son hasta "hagiográficos". Es verdad que leyendo ciertas frases se nota una vena de aproximación optimista respeto a los progresos de la joven nación: "la nuestra nacionalidad cuenta con 7 años de vida; y estos 7 años [...] nos han llevado un ejército buenísimo, una notable marina, un sistema de ferrocarriles que conecta todas las partes de Italia, y facilita el juntarse entre las poblaciones y sus intereses” (Trivulzio 1868: 71).

\footnotetext{
${ }^{3}$ Para profundizar el pensamiento político e ideológico de la princesa, véase Trivulzio di Belgiojoso 1847, 1848, 1866, 1868, 1904, 1977, 1978, 1993, 1997, 1998.

4 Todas las traducciones del ensayo analizado en el artículo son mías.
} 
En esta obra, la escritora insiste mucho en un pasado que fue época de esclavitud y en cómo que un pueblo "educado a la esclavitud", para transformarse en un pueblo nuevo, tenga que lograr combatir a "las tendencias que se oponen al desarrollo moral, intelectual y nacional" (Trivulzio 1868: 75). Maldini Chiarito, que se muestra bastante critica con algunos aspectos de la obra, destaca que entre "valoraciones personales y pedantes y esquemáticos complacimientos de los resultados alcanzados afloran también algunas incisivas afirmaciones sobre la importancia y la urgencia del fare gli italiani" (Maldini Chiarito 2010: 217). La urgencia de crear una serie de valores compartidos por toda la población y unas altas aspiraciones sociales y políticas. Ahora que la nación estaba unificada había que crear una escalera de valores positivos y educar los ciudadanos a ellos.

Sin embargo, son muchas las temáticas que Cristina trata en su ensayo político y que se pueden analizar por separado, entre ellas el victimismo y la incapacidad de los ciudadanos, que creen que no pueden cambiar su realidad: "una plaga dejada por el despotismo extranjero es la inclinación a imputar cada daño, cada desventura, cada calamidad al gobierno" (Trivulzio 1868: 128). Culpar al gobierno de los males sociales, sin hacer realmente nada para erradicarlos, es evidentemente una arraigada costumbre italiana. Para combatir esa tendencia, Cristina propugna el activismo y la ejemplaridad, porque quién ejemplifica gana tanto en credibilidad como en autoridad. A su juicio, no existen excusas para que el individuo no se comprometa con un profundo cambio social:

Se me contestará quizás que todo individuo, por muy operoso y deseoso del bien que sea, nada puede contra la multitud. Yo, en cambio, creo que cualquiera, por débil que sea naturalmente, adquiere una admirable autoridad sobre las masas, cuando camina a cara descubierta y con la frente alta en la justa dirección [...]. Hoy las asociaciones de los ciudadanos son, no solo permitidas, sino recomendadas y protegidas: por eso ningún individuo puede disculpar su propia inacción con el pretexto de que le está denegado actuar. (Trivulzio 1868: 130)

Recordando el despotismo y el absolutismo de quién gobernó antes de la unificación, los austriacos en el Norte y los borbones en el Sur, se evitan otros males tales como la indiferencia o la inercia. La voz, no coral, de Cristina, advierte a los italianos: 
Observen los hombres juiciosos de cada ciudad, de cada provincia italiana, los efectos funestos que produjo en la ciudadanía el despotismo extranjero. Digan a quién lo desconoce, que la libertad y la independencia de una nación, ya esclava de la caída del imperio romano hasta hoy, no son bienes que se adquieren con poco gasto y poco esfuerzo; [...] Y mientras enseñan a quienes ignoran las primeras y más básicas verdades fundamentales de la vida nacional y civil, empéñense en remediar de alguna forma los daños reales que causan descontento en el pueblo. (Trivulzio 1868: 131)

Una solución al descontento y a la desigualdad, es el asociacionismo de la ciudadanía. Es muy interesante leer como Cristina promueve la cooperación de los ciudadanos, de los pequeños empresarios y de los artesanos, e invoque la creación de bancos populares que ayuden al pequeño crédito en contra de los grandes capitalistas. Sus palabras son de una actualidad sorprendente:

[que] se abran unos negocios cooperativos, los bancos populares, y otras instituciones similares, para combatir las maquinaciones de ciertos capitalistas, que se enriquecieron especulando a costa de la pobreza y de la ignorancia del pueblo. En conclusión, yo querría que se formara en Italia una amplísima asociación, en la cual se inscribiesen todos los hombres dotados de sentido común, de patriotismo y de honestidad para poner en común sus facultades, sus medios y sus pensamientos al objeto de liberar al pobre de su miseria. (Trivulzio 1868, 131)

Trivulzio desea que Italia sea una nación solidaria, en la que los más privilegiados, como ella, culta y rica, se preocupen por los más desfavorecidos sin manifestar una actitud elitista o conservadora y se comprometan con la recuperación social de los marginados, incluso los que están al margen de la justicia:

Y hasta que tan inmensa asociación no sea formada [...], querría que los [...] más activos, expertos y cultos de las ciudades de Italia se uniesen y formasen unas asociaciones parciales, tendientes todas al mismo objetivo, sin olvidar que al mismo tiempo deben intentar, también como simples individuos, persuadir a los ignorantes y corruptos para que cesen en sus errores dado el daño que a ellos y al país entero ocasionan [...] Mientras esta idea esté siempre presente, habré obtenido el fin que me prefije escribiendo estas hojas: que no falten medios a quienes los buscan con el firme propósito de emplearlos. (Trivulzio 1868: 132) 
Cristina traslada su visión del conflicto social tanto al cercano contexto europeo como al más amplio contexto mundial. A su juicio, se puede defender la libertad del pueblo italiano, su independencia, sin recurrir al belicismo o a la xenofobia, porque "para que una nación pueda decirse, de buena ley, independiente, no es necesario que rechace toda influencia extranjera" (Trivulzio 1868: 133).

Ella considera que entre los patriotas italianos que han trabajado activamente por la unificación, como ella, y que disfrutan de un escaño en el parlamento, a diferencia de ella, por ser varones, se cuentan muchos ultra liberales que nada tienen que ver con los valores democráticos:

Estos fanáticos de la independencia querrían que la nación caminase siempre en la dirección que más desagrada, más ofende, o más amenaza a las naciones más cercanas. El chocar voluntariamente contra quién no ha provocado ofensa, no es un acto de independencia, sino de absurda arrogancia y de no justificada prepotencia [...]. Una nación puede considerarse verdaderamente libre, cuando no pida obedecer solo las leyes que [...] hayan sido aprobadas por la mayoría de los representantes de la nación. (Trivulzio 1868: 134)

Cristina Trivulzio nos explica que su concepto de libertad está en total antítesis con ésos fanáticos:

La libertad como yo la entiendo sacrifica en cierta medida al individuo por la nación, y no considera aquello si no como parte integral o como representante de esta. La libertad como le entienden los ultras liberales, la libertad indefinida y no definible, no confiesa la necesidad de sacrificar al individuo por la nación, ni la nación por el individuo, sino que sacrifica ambos por una ilusión, una falsa doctrina. (Trivulzio 1868: 135)

En la Historia reciente, la palabra "libertad" ha sido utilizada inoportunamente por personajes muy antiliberales, protagonistas de las políticas internacionales. Trivulzio demuestra, además, en este ensayo, ser una brillante analista económica. Se queja de un problema que es todavía muy actual en Italia, el problema de la dependencia energética, pensamos en el gas que se importa de Rusia, o en la energía eléctrica que viene de Francia. "De muchos elementos de prosperidad carece, sin embargo, Italia -escribe- la falta 
considerada hasta aquí irremediable de carbón fósil, y el elevado precio al cual tenemos que adquirirlo en regiones lejanas" (Trivulzio 1868: 136-137).

Cristina explica cómo la aceptación por parte de Italia de un liberalismo desenfrenado y el abandono de cualquier tipo de tutela proteccionista en el ámbito del mercado afecta gravemente el mercado interno:

Y este obstáculo al progreso de las industrias metalúrgicas es altamente perjudicial para las demás industrias porque obliga a adquirir en el exterior distintas e innumerables maquinas [...] esto es así por haber rechazado el sistema comercial protector, como tiránico y opresor, y por haber adoptado en su lugar el principio del libre comercio. De hecho, la facultad, concedida a todas las naciones, de poder mandar sus productos industriales a Inglaterra, sin pago de impuesto alguno, implicando naturalmente para Inglaterra un derecho reciproco, permite que esta sea dueña y señora de todo el mercado exterior, y que que lo invada con productos industriales superiores. Nadie, ni siquiera los mismos italianos, aceptan productos italianos, imperfectos, poco duraderos, carísimos. (Trivulzio 1868: 137)

Cristina es una mujer con una gran consciencia social, y vuelve al tema de la importancia del ejemplo. Es consciente de que su condición socio-cultural le permite tener esa actitud de compromiso hacia las instituciones:

Pero somos guías silenciosos y maestros intolerantes; hacemos maravillas porque el ardor de quién sigue no se sostiene como el nuestro, olvidando que la expectativa de futuro, la cual alimenta nuestra constancia, no consuela a las multitudes. Nosotros toleramos con buena voluntad las privaciones y los sacrificios, porque vemos el termino, y sabemos cuáles serán los resultados; pero el pueblo lo ignora, y cuando nos ve caminar delante, e invitarlo a seguirnos. (Trivulzio 1868: 138)

Cristina entiende al pueblo desanimado frente a los problemas de la nación con verdadera empatía e identificación, insistiendo en hacer hincapié en la importancia de ser creíble:

Acordémonos, entonces, que las masas no pueden continuar apegadas a un orden de cosas que no les proporciona ningún beneficio material y que no les permite albergar esperanza alguna de futuras ventajas. Esforcémonos en mejorar la suerte de las clases más pobres de nuestras poblaciones en tanto no se efectúa una mejora que sea perceptible por ellas. (Trivulzio 1868: 139) 
Cristina fomenta y anima a la cooperación de la pequeña y mediana empresa contra el gran capital, que explota los trabajadores pagándoles mal. Se expresa con palabras muy actuales y aclama:

La asociación de los capitales, de los elementos industriales y de los artesanos que proveen al comercio con los productos de su industria. El principal objetivo de esa asociación sería suprimir las gastos superfluos y los deshonestos beneficios de los que hoy disponen de los capitales, que dirigen la industria con el único fin de enriquecerse a sí mismos, engañando a los compradores, a los cuales dispensan mercancías averiadas y defectuosas, no concediendo al pobre artesano nada más que aquella mínima paga que basta para sostener míseramente la vida, para luego abandonarlo a la caridad de los hospicios y de los lugares de refugio, cuando la juventud y la fuerza se agotan. (Trivulzio 1868: 140)

Otro eje fundamental de la visión política de Trivulzio es la emancipación de las clases más desfavorecidas, para ello, es fundamental fomentar el valor de la educación en sentido genérico y el de la educación en derechos de modo específico como punto de partida para la defensa contra la explotación laboral:

Para resumir, diré que el objetivo hacia al cual tenemos que tender por primero, es difundir la luz en las mentes de las clases pobres de nuestras poblaciones, para hacerlas conscientes de sus derechos, y darles los medios para escapar de las garras sangrientas de los expoliadores de todo tipo que hoy los tienen alienados. (Trivulzio 1868: 141)

Una educación que, para Cristina, debe ser necesariamente laica. Pese a que es una mujer religiosa, invoca la laicidad de la sociedad como medio fundamental para combatir la ignorancia de las masas y su predisposición a la manipulación:

Repetimos siempre que nuestras poblaciones agrícolas y artesanas están en las manos del clero, que las educa a su manera aprovechando su ellas creen ciegamente en él: sabemos que la mayoría del clero ve con malos ojos, y además vitupera y condena, todo lo que se ha hecho en Italia desde en el "59 en adelante, y no hacemos nada para evitar que el clero se apropie de las mentes y los corazones de nuestras poblaciones [...]. ¿De quién es, entonces, la culpa, si nuestro pueblo está tan poco informado en relación a las reglas fundamentales de la convivencia civil? (Trivulzio 1868: 142-143) 
La necesidad de extender el derecho a la educación a las masas más pobres es fundamental, dice la autora, sin embargo, hay que exigirse a sí mismo una moralidad incorruptible, hay que ser modélico para estar en condiciones de demandar un cambio en las masas populares:

Esforcémonos en inspirar a nuestros compatriotas, con independencia de su clase social, la tolerancia, la constancia y la energía. Erradiquemos las tinieblas de la ignorancia que priva al pobre de los campos y al de las ciudades de la necesaria luz; pero mientras educamos al pobre, no olvidemos educarnos a nosotros mismos. Tenemos que erigirnos en maestros del pueblo, corrigiendo nuestros defectos para que pueda ver en nosotros el modelo de ciudadanía propio de un país libre. (Trivulzio 1868: 143)

Cristina termina este testamento político invitando a la ciudadanía a romper con la inercia generalizada: que "cada uno haga lo que sepa y pueda hacer, y [que] juzgando su actitud no se deje engañar por el amor al ocio, sino que haga de sí un serio y escrupuloso examen -escribe-. Estos son los resultados hacia los cuales todos tenemos que tender, en la medida de nuestras fuerzas y de nuestra capacidad" (Trivulzio 1868: 143). Volver a descubrir el pensamiento político de Cristina Trivulzio di Belgiojoso no es solo fundamental para tener una visión completa de su obra artística y de su experiencia vital o para poder apreciar su coherencia y su valor; también es relevante como fuente de inspiración, si bien incómoda, para la actual clase política italiana.

\section{Referencias bibliográficas}

AA.VV., 1883. La Centuria di donne illustri italiane. Milano: Biblioteca del popolo.

Angeloni, Vincenzo. 1911. Le donne nel Risorgimento italiano. Venezia: Emporio.

Barbiera, Raffaello. 1923. Italiane gloriose. Milano: Vallardi.

1902. La principessa Belgiojoso. I suoi amici e nemici, il suo tempo. Milano: Treves.

1903. Passioni del Risorgimento. Nuove pagine sulla principessa Belgiojoso e il suo tempo. Milano: Fratelli Treves. 
Cepeda Fuentes, Marina. 2011. Sorelle d'italia. Le donne che hanno fatto il risorgimento. Torino: Blu.

Comba, Eugenio. 1935. Donne italiane illustri. Torino: Paravia.

Gasparinetti, Anna. Quattro anni di attività giornalistica della principessa Cristina Trivulzio di Belgioioso (1845-48). "Rassegna storica del Risorgimento", a. XVII, fasc. I, gennaio-marzo 1930. 72 - 104.

Gioberti, Vincenzo. 1911. Del rinnovamento civile d'italia, Bari: Laterza.

Luzio, A, in Cristina Belgioioso, in "Il corriere della Sera", Milano, 3 agosto 1902.

Maldini Chiarito, Daniela, in Gli scritti della vecchiaia in AA.VV., 2010. La prima donna d'italia. Cristina Trivulzio di Belgiojoso tra politica e giornalismo. Roma: Franco Angeli.

Malvezzi, Aldobrandino. 1936. Cristina di Belgioioso. Milano: F.lli Treves.

Rossi, Mino. 2005. Cristina Trivulzio, principessa di Belgiojoso. Il pensiero politico. Passirano (Br): Franciacorta.

Severgnini, Luigi, 1972. La principessa di Belgiojoso, Milano: Virgilio.

Trivulzio di Belgiojoso, Cristina. 1847. Studi intorno alla storia della Lombardia negli ultimi trent"anni e delle cagioni di difetto d'energia dei Lombardi. Parigi: S.n.t..

5 maggio del 1848. "L'organizzazione della Guardia Nazionale in Lombardia". Il crociato.

. 1866. "Della presente condizione delle donne e del loro avvenire". Nuova antologia di scienze, lettere ed arti, v. 1: 96-113.

1868. Osservazioni sullo stato attuale dell'Italia e sul suo avvenire. Milano: Francesco Vallardi.

1904. L'Italia e la rivoluzione italiana (dalla Revue des Deux Mondes, 1848) aggiuntovi gli ultimi tristissimi fatti di Milano, narrati dal Comitato di pubblica difesa, con documenti. Palermo: Sandron.

. 1977. Il 1848 a Milano e Venezia con uno scritto sulla condizione delle donne, a cura e con traduzione di Sandro Bortone. Milano: Feltrinelli.

. 1978. Ricordi dall'esilio. Cinisello Balsamo: Edizioni Paoline.

. 1993. Vita intima e vita nomade in Oriente. Como: Ibis.

1997. Emina. Ferrara: Tufani.

1998. Un principe curdo. Ferrara: Tufani. 
2011. Cristina Trivulzio di Belgioioso. De la presente condición de las mujeres y de su futuro [ed. Mercedes Arriaga Florez]. Sevilla: Arcibel. 